\title{
Mephobarbital Measurement
}

National Cancer Institute

\section{Source}

National Cancer Institute. Mephobarbital Measurement. NCI Thesaurus. Code C75366.

The determination of the amount of mephobarbital present in a sample. 